\title{
JOGOS EDUCACIONAIS COMO ESTRATÉGIA PEDAGÓGICA DE ENSINO DOS CONTEÚDOS ESCOLARES
}

\author{
Apresentação: Comunicação Oral
}

\author{
Noel Gomes da Silva Filho ${ }^{1}$ \\ Albertina Marília Alves Guedes² (Orientadora)
}

\begin{abstract}
Resumo: De acordo com alguns estudiosos da área de educação e tecnologia é importante que na contemporaneidade os professores utilizem, quando possível, ferramentas e/ou recursos tecnológicos visando mediar os conteúdos curriculares de modo mais divertido e interativo com os instrumentos tecnológicos. Esse trabalho foi realizado no $1^{\circ}$ semestre de 2016 e teve colaboração de uma professora da disciplina de Biologia e 13 estudantes do $1^{\text {a }}$ ano do Ensino Médio de uma escola pública estadual localizada na cidade de Petrolina/PE e objetivou apresentar aos alunos softwares e jogos educacionais que podem ser utilizados no processo de aprendizagem dos conteúdos escolares e viabilizar o uso de jogos educativos no processo de ensino e aprendizagem dos conteúdos escolares como apoio didático. Os instrumentos utilizados na coleta de dados foi observação participante durante as aulas da professora de biologia, notebook com acesso à internet para vista ao site do NOAS. Em seguida, agendamos um horário conveniente a todos os participantes um momento na Biblioteca da escola para explicar aos participantes sobre a pesquisa, os objetivos, os conteúdos abordados e formas de coleta de dados. Posteriormente, os alunos foram convidados a participarem de uma entrevista sobre o método de ensino mediante o uso de instrumentos tecnológicos. Os dados coletados foram analisados a partir de uma abordagem qualitativa e analisados com embasamento na Análise de Conteúdo. Os resultados desta pesquisa revelaram que os alunos demonstraram interesse, motivação e maior participação durante os momentos de socialização dos conteúdos. Os alunos ainda relataram que gostariam de dar continuidade ao método de aprendizagem por intermédio de jogos educacionais. Por fim, diante destes resultados percebemos que o uso de ferramentas e/ou instrumentos tecnológicos para fins pedagógicos tem um papel importante na educação e na construção do conhecimento por parte dos alunos.
\end{abstract}

Palavras-Chave: Jogos Educacionais, Estratégias Pedagógicas, Ensino, Aprendizagem.

\section{INTRODUÇÃO}

Atualmente a tecnologia está cada vez mais presente em diversos lugares gerando uma maior influência na sociedade e, consequentemente, no ambiente escolar. No contexto escolar professores e alunos estão cercados de ferramentas tecnológicas que podem ser utilizadas como um instrumento de ensino e aprendizagem. De acordo com alguns teóricos, mediante o uso de instrumentos tecnológicos no ambiente escolar o aluno pode se sentir mais motivado pelos conteúdos escolares (MORAN, MASETTO e BEHREMS, 2013).

\footnotetext{
${ }^{1}$ Estudante do Curso de Licenciatura em Computação, IF Sertão PE, E-mail: noelgomesdasilvafilho@gmail.com ${ }^{2}$ Professora do Curso de Licenciatura em Computação, IF Sertão PE. E-mail: albertina.guedes@ifsertao-pe.edu.br
} 
Na concepção de Moran, Masetto e Behrens (2013), os jogos educacionais que estão cada vez mais ganhando espaço no contexto escolar como estratégia de ensino dos conteúdos curriculares. Além disso, Moran, Masetto e Behrens (2013) ainda ressaltam que muitos professores tem obtido êxito em mediar os conteúdos escolares a partir da realização de jogos educativos sobre os conteúdos curriculares realizados por intermédio de instrumentos tecnológicos. Para esses autores, realizar esse tipo de atividade pedagógica tem sido benéfico visto que tem influenciado de modo significativo no processo de aprendizagem dos estudantes em sala de aula.

A partir deste pressuposto esse trabalho tem como problema fomentar uma discussão sobre os desafios e possibilidades de inserir nas atividades pedagógicas desenvolvidas em sala de aula mediante a inserção de jogos educacionais acerca dos conteúdos escolares como estratégia pedagógica que viabiliza uma melhor aprendizagem por parte dos alunos. A realização desta pesquisa foi considerada relevante visto que, na atualidade, muitos alunos tem fácil acesso aos instrumentos tecnológicos, principalmente aparelhos celulares com acesso à internet e, visando elevar a qualidade do processo de aprendizagem dos estudantes, é importante que os professores saibam aliar o uso de instrumentos tecnológicos objetivando elevar a qualidade e o interesse dos alunos pelos conteúdos escolares.

Esse trabalho foi realizado com a colaboração de uma professora da disciplina de Biologia e 13 estudantes do $1^{\text {a }}$ ano do Ensino Médio de uma escola pública estadual localizada na cidade de Petrolina/PE. Os dados coletados foram analisados a partir de uma abordagem qualitativa e, por isso, neste trabalho não teremos uma hipótese pré-concebida sobre os resultados esperados com a coleta dos dados. Esse estudo tem como principais objetivos: 1) Apresentar aos alunos softwares e jogos educacionais que podem ser utilizados no processo de aprendizagem dos conteúdos escolares, e, 2) Viabilizar o uso de jogos educativos no processo de ensino e aprendizagem dos conteúdos escolares como apoio didático.

\section{FUNDAMENTAÇÃO TEÓRICA}

$\mathrm{Na}$ atualidade a tecnologia vem se tornando cada vez mais comum nas escolas, quer seja através de um Laboratório de Informática com computadores ou por intermédio do uso de celulares e/ou notebooks em sala de aula por parte de professores e alunos. Conforme apresentado por Moran, Masetto e Behrams (2013), essa mesma tecnologia veio para modificar o modo tradicional de ensino possibilitando a professores uma nova forma de ensinar e aos alunos um meio diferente de aprender os conteúdos escolares ministrados em sala de aula.

Diante desta nova realidade tecnológica disponível, Moran, Masetto e Behrams (2013, p. 78) apresentam que muitas pessoas, tem fácil acesso aos instrumentos tecnológicos e, por isso, “o 
professor precisa refletir e realinhar sua prática pedagógica no sentido de criar possibilidades para instigar a aprendizagem do aluno”. Esses teóricos ainda apresentam que o uso de instrumentos e/ou ferramentas tecnológicas em sala de aula pode ser compreendido por professores como sendo uma possibilidade de viabilizar o ensino dos conteúdos escolares de modo mais prazeroso e interativo. Além disso, é capaz de incentivar o aluno a buscar conhecimento, expandindo os horizontes na busca por conhecimentos para além da escola. Assim sendo, uma destas tecnologias que iremos abordar neste trabalho será o uso de jogos educacionais como possibilidade de estratégia de ensino.

Sobre o uso de instrumentos tecnológicos processo de ensino e aprendizagem de conteúdos escolares Falkembach (2013, p. 1) apresenta que “os jogos educacionais computadorizados são softwares que apresentam conteúdo e atividades práticas com objetivos educacionais baseados no lazer e diversão". Assim sendo, podemos dizer que esses tipos de jogos educacionais são desenvolvidos com o principal objetivo de contribuir com as atividades didáticas executadas por professores em sala de aula. Assim, através de conteúdos e atividades bem elaboradas que estejam presentes nos jogos, os estudantes são incentivados a estudar e a pesquisar tudo que lhes é ensinado, fazendo do jogo um aliado para à educação.

Na concepção de Aragão e Nez (2010), é possível tornar os jogos educacionais uma ferramenta didática atuando no auxílio de uma aprendizagem mais prazerosa e motivadora por parte dos alunos. Por outro lado, Batista e Dias (2012) apresentam que os jogos ajudam na aprendizagem e se tornam uma disputa com diversão para o caminho do saber quando são usados como instrumentos de ajuda. Logo podemos perceber que quando o professor faz uso deste tipo de estratégia pedagógica de ensino mediante o recurso de instrumentos tecnológicos viabiliza o processo de aprendizagem mais interativo e motivador.

Prensky (2012) ainda ressalta que a aprendizagem baseada em jogos digitais viabiliza que o professor explore ao máximo os conteúdos ministrados em sala de aula estimulando nos alunos maior aprendizagem. Partindo desse conceito, é possível compreender que alguns conteúdos ministrados a partir do modelo tradicional de ensino a educação por mais desestimulantes que sejam para alguns, podem ser aprendidos através do uso de jogos educacionais e de forma divertida (SAVI e ULBRICHT, 2008).

De acordo com Batista e Dias (2012), uma vantagem em utilizar jogos educativos consiste em induzir o aluno a perceber que, a partir do momento em que o aluno entra em contato com um novo conhecimento através de jogos pedagógicos ele pode perceber que existem outras maneiras de aprender além do modelo tradicional, em consequência disso, é possível que o aluno desperte o interesse e motivação pelos conteúdos escolares. 
Sobre o uso da internet e dos instrumentos tecnológicos em sala de aula Moran (2009, p. 11) ainda ressalta que: "a internet é uma tecnologia que facilita a motivação dos alunos, pela novidade e pelas possibilidades inesgotáveis de pesquisa que oferece". Para Moran (2009), a internet pode estimular a motivação pela aprendizagem porque contribuição na formação de laços afetivos entre o professor, o aluno e o conteúdo a ser estudado em sala de aula. Além disso, também pode facilitar o processo de ensino aprendizagem devido à capacidade de comunicação que o professor desenvolve e estabelece com o aluno.

Todavia, Moratori (2003), enfatiza que para uma utilização eficiente e completa de um jogo educativo computadorizado é necessário realizar uma análise consistente sobre o mesmo, verificando tanto aspectos pedagógicos como aspectos de qualidade de software. É importante o destaque dado por Moratori (2003) visto que nem todos os jogos indicados como educativos tem objetivos pedagógicos e, por isso, antes de aplicar esse tipo de jogo em sala de aula é preciso que o professor tenha um prévio planejamento sobre os conteúdos que serão abordados por intermédio do jogo, bem como os objetivos que se pretende alcançar ao fazer uso de determinado jogo.

\section{PROCEDIMENTOS METODOLÓGICOS}

Esse estudo diz respeito a uma pesquisa de campo de abordagem qualitativa do tipo descritiva realizada durante o período de abril a agosto de 2016 (GERHARDT e SILVEIRA, 2009). Os participantes dessa pesquisa foram 13 estudantes do $1^{\text {a }}$ ano do Ensino Médio da Escola Estadual Humberto Soares, localizada na zona urbana da cidade de Petrolina-PE. A coleta de dados foi realizada durante 15 minutos após o término das atividades dos encontros realizados na Biblioteca da própria escola e durante as aulas da disciplina de Artes.

Visando ter uma melhor compreensão sobre a temática discutida neste estudo foi realizado uma discussão teórica sobre quais podem ser as possíveis contribuições do uso de instrumentos e/ou recursos tecnológicos no processo de ensino e aprendizagem da disciplina de ciências e biologia. Objetivando obter dados qualitativos para a pesquisa foi realizado, mediante a permissão da professora de biologia e os estudantes do $1^{\circ}$ ano do Ensino Médio, observação das aulas de biologia. Após os momentos de observação em sala de aula foi feito o convite para os alunos que tivessem interesse de participar da pesquisa como voluntários.

Para a coleta de dados, foi realizada a apresentação do projeto aos participantes, bem como os objetivos. Nos encontros seguintes, foram explicados os assuntos que seriam trabalhados durante o projeto mediante o uso do netbook e acesso à internet por intermédio dos jogos sobre os conteúdos de biologia disponíveis no site do Núcleo de Computação Aplicada - NOAS. 
O NOAS é destinado ao desenvolvimento de objetos de aprendizagem significativa, estruturados em simulações computacionais de fenômenos o qual foi criado com a proposta de desenvolver objetos que contribuam para uma aprendizagem significativa. Essas atividades são baseadas em simulações computacionais, tais como: applets java, animações em flash, realidade virtual, dentre outros recursos, que permitem ao aprendiz a interação necessária à compreensão dos fenômenos estudados. A equipe do NOAS é constituída por educadores e especialistas em softwares que fazem uso da tecnologia digital como elemento potencializador do processo de ensino e aprendizagem. Além disso, também há disponível no site uma biblioteca virtual com aplicativos educacionais de diversas áreas de conhecimento, conforme informações disponíveis no site: http://www.noas.com.br/.

Posteriormente, os alunos foram divididos em 3 sub-grupos e, em seguida, foram realizadas atividades pedagógicas abordando os conteúdos de biologia mediante o uso de netbooks e acesso à internet. Por fim, no último encontro foi aplicado um questionário para alunos visando avaliar se os mesmos puderam aprender os conteúdos de biologia apresentados por intermédio de jogos educativos de modo mais prazeroso e motivador. Visando não expor os alunos participantes os mesmos são identificados neste trabalho como Aluno 1, Aluno 2 e assim sucessivamente.

Após a coleta de dados foi realizada a análise e interpretação dos dados coletados. O procedimento utilizado foi embasado na perspectiva da Análise de Conteúdo de Bardin (2009).

\section{RESULTADO E DISCUSSÃO}

Na concepção de Bardin (2009), quando um pesquisador utiliza esse tipo de método para analisar dados de pesquisa de abordagem qualitativa, é possível descrever, analisar e interpretar os fenômenos observados no contexto da investigação. Além disso, para Bardin (2009), quando se busca coletar dados e informações num contexto de pesquisa, é importante que o pesquisador compreenda que não é possível encontrar dados e/ou informações prontas, todavia, existem algumas regras e critérios que possibilitam, ao pesquisador, alcançar os objetivos propostos em sua investigação. Assim sendo, os resultados obtidos durante a coleta de dados estão descritos abaixo conforme relatos dos estudantes participantes as questões realizadas durante as entrevistas.

A primeira questão realizada aos estudantes foi: $\mathrm{O}$ jogo realizado contribuiu para sua aprendizagem? Caso a resposta seja afirmativa cite um exemplo do que aprendeu. Abaixo segue as respostas dos entrevistados:

Sim, a prevenção, o grupo pertencente da doença, e qual o vetor - Aluno 5.

Sim, pois ajuda a desenvolver o aprendizado, porque a causa da doença e pude melhor 
que ocorre é através de um vírus - Aluno 8.

Sim, pois, como pude aprender, é importante a prevenção e conhecer o que a doença pode causar-Aluno 13.

De acordo com os entrevistados utilizar jogos na aprendizagem de conteúdos escolares, tais como, Biologia pode viabilizar uma aprendizagem mais significativa, interativa e divertida. Nas falas dos participantes ainda percebemos, em relação ao uso de jogo pedagógico sobre "Parasitologia" os entrevistados relataram que puderam compreender melhor o conteúdo estudado.

Esses dados confirmam o que Aragão e Nez (2010) apresentam sobre o modo como uso de jogos educativos podem contribuir no processo de aprendizagem e torná-la mais prazerosa e motivadora. Além disso, a partir desses dados percebemos também que o uso do jogo educativo de Biologia possibilita uma melhor aprendizagem. Além disso, conforme revela Savi e Ulbricht (2008) explicam sobre o uso do jogo com objetivos pedagógicos também pode viabilizar uma aprendizagem mais rápida e motivadora.

Os entrevistados também foram questionados sobre a metodologia de ensino executada em sala de aula por intermédio do jogo mediante a seguinte questão: "como considera a experiência de executar atividades pedagógicas sobre conteúdos de Biologia a partir do uso de um software educativo?". Neste questionamento os participantes responderam:

Bom! Eu achei muito útil para a aprendizagem e também foi muito divertido aprender biologia com esse jogo - Aluno 5.

Eu achei muito legal. Eu nunca tinha jogando um jogo como esse. A aprendizagem é mais interessante. Gostei muito - Aluno 9.

Foi bom. Aprender com o uso deste jogo torna a aula diferente e divertida-Aluno 11.

Os relatos dos entrevistados demonstram que o uso de jogos pedagógicos no ensino de conteúdos pedagógicas além de contribuir na aprendizagem dos alunos ainda pode ser concebido como um momento de diversão e despertar maior interesse dos estudantes pelo conteúdo ministrado pelo professor, conforme descrito por Falkembach (2013).

Sobre o uso do jogo utilizado sobre conteúdos de "Parasitologia", Falkembach (2013) ainda ressalta que o referido software pedagógico pode ser utilizado pelo professor como um instrumento tecnológico que visa romper com o modelo tradicional de ensino, e, por isso, pode despertar o interesse dos alunos pela aprendizagem. Todavia, Prensky (2012) destaca que o professor deve 
previamente elaborar um bom planejamento pedagógico com objetivos claros acerca de que conteúdo será abordado através do jogo.

Por fim, questionamos se os alunos tinham interesse em dar continuidade às atividades pedagógicas de ensino e aprendizagem dos conteúdos da disciplina de Biologia mediante o uso de jogos pedagógicos. Abaixo segue as respostas dos participantes:

Tenho interesse sim, pois achei bem divertido aprender com o jogo. Quero sim dar continuidade e aprender com jogos - Aluno 3.

Tenho interesse em dar continuidade a participar dos jogos para aprender Biologia. $O$ jogo ajuda na aprendizagem e é bom pra aprender mais assuntos - Aluno 12.

A partir das respostas acima dos alunos, percebemos que, em relação a problemática apresentada sobre os desafios e possibilidades de inserção dos jogos em atividades pedagógicas desenvolvidas em sala de aula é possível despertar o interesse e motivação dos alunos pela aprendizagem mediada pelo uso de instrumentos tecnológicos, tais como softwares pedagógicos que viabilizam a aprendizagem a partir da interação do aluno com um jogo (PRENSKY, 2012; BATISTA e DIAS, 2012).

A seguir apresentamos na Imagem 1 a página do site do NOAS que disponibiliza os jogos da disciplina de Biologia sobre "Parasitologia". Na Imagem 2, apresentamos alguns dos momentos da realização das atividades sobre o conteúdo de "Parasitologia" com os estudantes sobre o uso de jogos pedagógicos disponível no site do NOAS.

Imagem 1: Interface do jogo Parasitologia. Fonte: Própria

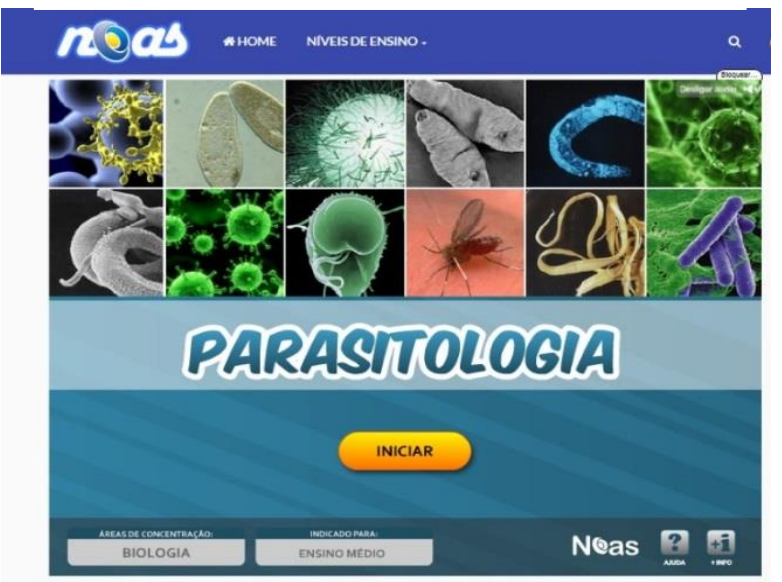

Imagem 2: Alunos jogando através de netbooks do colégio. Fonte: Própria.

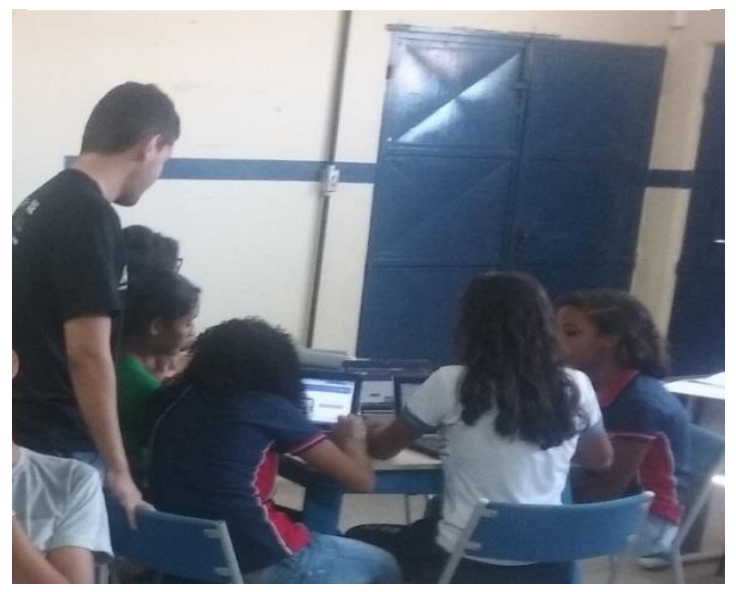




\section{CONSIDERAÇÕES FINAIS}

Com a realização deste trabalho percebemos que o jogo educacional pode viabilizar aprendizagem e interação dos alunos com os instrumentos tecnológicos. Além disso, possibilita também que os alunos possam aprender de modo diferente do modelo tradicional de ensino. No entanto, é preciso que o professor faça um bom planejamento pedagógico sobre o modo como o conteúdo será abordado em sala de aula.

Esse estudo também revelou contribuir para a discussão sobre o uso de jogos educacionais executados mediante o uso de instrumento tecnológicos, tais como: computador e/ou tablet, softwares educacionais e internet, viabilizando o uso de jogos como apoio didático no processo de ensino e aprendizagem. A partir do uso desses recursos os estudantes participantes demonstraram interesse nas aulas desenvolvidas com jogos e indicando sucesso em realização de tal aula com essa ferramenta, evidenciando assim o que relatam os teóricos citados neste trabalho.

Por fim, diante destes resultados percebemos que o uso de ferramentas e/ou instrumentos tecnológicos para fins pedagógicos tem um papel importante na educação e na construção do conhecimento por parte dos alunos.

\section{REFERÊNCIAS}

ARAGÃO, D. S; NEZ, E. Jogos Educativos com o uso do Computador na Educação Infantil. II Simpósio Nacional de Educação e XXI Semana de Pedagogia. Infância Sociedade e Educação. Universidade Estadual do Oeste do Paraná - UNIOESTE, Campus de Cascavel, 13 a 15 de Outubro de 2010.

BARDIN, L. Análise de Conteúdo. Lisboa: LDA, 2009.

BATISTA, D. A; DIAS, C. L. O Processo de Ensino e de Aprendizagem Através dos Jogos Educativos no Ensino Fundamental. Anais do Encontro de Ensino, Pesquisa e Extensão. Colloquium Humanarum, v. 9, Edição Especial, jul-dez, p. 975-982. Presidente Prudente, SP, 22 a 25 de outubro, 2012,

FALKEMBACH, G. A. M. O Lúdico e os Jogos Educacionais. Trabalho de Conclusão de Curso. Curso de Especialista em Mídias na Educação. Centro Interdisciplinar de Novas Tecnologias na Educação da Universidade Federal do Rio Grande do Sul - UFRGS. Porto Alegre, 2013.

GERHARDT, T. E; SILVEIRA, D. T. (Org.). Métodos de Pesquisa. Porto Alegre: Editora da UFRGS, 2009.

MORAN, J. M. Novas Tecnologias e Mediação pedagógica. 16ª Ed. Campinas: Papirus, 2009.

MORAN, J. M; MASETTO, M; BEHRENS, M. Novas tecnologias e mediação pedagógica. Campinas: Papirus, 2013. 
MORATORI, P. B. Porque Utilizar Jogos Educativos no Processo de Ensino e Aprendizagem? Trabalho de Conclusão da Disciplina de Introdução a Informática na Educação. Mestrado de Informática Aplicada à Educação. Núcleo de Computação Eletrônica da Universidade Federal do Rio de Janeiro - UFRJ, Rio de janeiro, 2003.

PRENSKY, M. Aprendizagem Baseada em Jogos Digitais. São Paulo: Editora SENAC, 2012.

SAVI, R; ULBRICHT, V. R. Jogos Digitais Educacionais: benefícios e desafios. Revista Novas Tecnologias na Educação. Universidade Federal do Rio Grande do Sul, v. 6, n. 2, Dezembro, 2008. 